\title{
Thanks to all those who reviewed for Trials in 2015
}

Douglas G. Altman ${ }^{1 *}$, Curt D. Furberg ${ }^{2}$ and Jeremy M. Grimshaw ${ }^{3}$

\section{Contributing reviewers}

A peer-reviewed journal would not survive without the generous time and insightful comments of the reviewers, whose efforts often go unrecognized. Although final decisions are always editorial, they are greatly facilitated by the deeper technical knowledge, scientific insights, understanding of social consequences, and passion that reviewers bring to our deliberations. For these reasons, the Editors-in-Chief and staff of Trials warmly thank the 854 reviewers whose comments helped to shape the journal, for their invaluable assistance with review of manuscripts in Volume 16 (2015).

\author{
Isabel Adeyemi \\ UK
}

Rakesh Aggarwal

India

Vineet Ahuja

India

Bafeta Aïda

France

Rubens Albuquerque

Brazil

Rawan AlHeresh

USA

Kelli Allen

USA

Peter Allmark

UK

Terje Alraek

Norway

Cinthia Amorim

Brazil

\author{
Karin Amrein \\ Austria
}

Guipeng An

China

Martin Anders

Czech Republic

Susan Andersen

Denmark

Joachim Andrassy

Germany

Sharon Andrew

UK

Gavin Andrews

Ane Appelt

Denmark

Yvan Arlettaz

Switzerland

Nigel Armfield

Australia

\author{
Joanna Armstrong Schellenberg \\ UK

\section{Jeffrey K Aronson} \\ UK
}

Bérengère Aubry-Rozier

Switzerland

\section{Susan Aucott \\ USA}

\section{Karen Austrian \\ Kenya}

\section{Cristina Avendaño}

Spain

\author{
Nassim Ayoub \\ Germany \\ Samy Azer \\ Saudi Arabia

\section{Simon Bacon} \\ Canada
}

\section{George Bagias}

Greece

\footnotetext{
* Correspondence: doug.altman@csm.ox.ac.uk

${ }^{1}$ Centre for Statistics in Medicine, University of Oxford, Botnar Research

Centre, Windmill Road, Oxford OX3 7LD, UK

${ }^{2}$ Division of Public Health Sciences, Wake Forest University School of

Medicine, Medical Center Boulevard, Winston-Salem 27157-1063, NC, USA

${ }^{3}$ Clinical Epidemiology Programme, Ottawa Hospital Research Institute and

Department of Medicine, University of Ottawa, 501 Smyth Road, Ottawa K1H

8 L6, ON, Canada
} 
Ivan Bajsic

Slovenia

Liz Baker

UK

Christine Baldwin

UK

Karla Ballman

USA

Joyce Balls-Berry

USA

Carlos Bandeira de Mello

Brazil

Saber Davide Barbar

France

Alexandre Barbosa

Brazil

Karen Barker

UK

Pilar Barnestein-Fonseca

Spain

Rubina Barolia

Pakistan

Partha Basu

India

Erika Baum

Germany

Annette Becker

Germany

Yves Becouarn

France

David Bekelman

USA

Robert Bell

UK

M Fernanda Bellolio

USA

Eduardo Bergel

Argentina

Vance Berger

USA

Donna Berry

USA

James Betts

UK
Zhaoxiang Bian

Hong Kong

Nina Biehal

UK

Nerea Bielsa

Spain

Egbert Biesheuvel

Netherlands

Eveline Bijlard

Netherlands

Laurent Billot

Australia

Ariella Binik

UK

Bojan Biocina

Croatia

Bernadette Biondi

Italy

Gill Black

South Africa

Martin Blakemore

UK

Amy Blakemore

UK

Jane Blazeby

UK

Charlotte Blease

Ireland

Christian Blickem

UK

Jonathan Boote

UK

Bijan Borah

USA

Mark Borthwick

UK

Emmanuel Boselli

France

Catherine Bouman

Netherlands

Isabelle Boutron

France

Peter Bower

UK
Julia Boyle

UK

Tonje Braaten

Norway

Patrick Brady

USA

Marco Braggion

Italy

Mantaj Brar

Canada

Hugues Brat

Switzerland

Sanjin Braut

Croatia

Aline Brennan

Ireland

Graham Brennan

UK

Daniel Bressington

Hong Kong

Ivan Bristow

UK

Laurent Brochard

Canada

Anna Brokmeier

Germany

Marie Brossar-Racine

USA

Deborah Brown

UK

Sarah Brown

UK

Iris Brunner

Norway

Deborah Buck

UK

Dario Bugada

Italy

Lorraine Buis

USA

Mark Bullimore

USA

Claudia Buntrock

Netherlands 


\begin{tabular}{|c|c|c|}
\hline $\begin{array}{l}\text { Matthew Burg } \\
\text { USA }\end{array}$ & $\begin{array}{l}\text { Annabel Chen-Tournoux } \\
\text { Canada }\end{array}$ & $\begin{array}{l}\text { Emilie Combet } \\
\text { UK }\end{array}$ \\
\hline $\begin{array}{l}\text { M Cairns } \\
\text { UK }\end{array}$ & $\begin{array}{l}\text { Stephen Chester } \\
\text { Australia }\end{array}$ & $\begin{array}{l}\text { Christian Compagnone } \\
\text { Italy }\end{array}$ \\
\hline $\begin{array}{l}\text { Robin Callister } \\
\text { Australia }\end{array}$ & $\begin{array}{l}\text { Alessandro Chiarotto } \\
\text { Netherlands }\end{array}$ & $\begin{array}{l}\text { Felix Compen } \\
\text { Netherlands }\end{array}$ \\
\hline $\begin{array}{l}\text { Michael Campbell } \\
\text { UK }\end{array}$ & $\begin{array}{l}\text { Dixon Chibanda } \\
\text { Zimbabwe }\end{array}$ & $\begin{array}{l}\text { Lynda Constable } \\
\text { UK }\end{array}$ \\
\hline $\begin{array}{l}\text { Marion Campbell } \\
\text { UK }\end{array}$ & $\begin{array}{l}\text { Wai Tong Chien } \\
\text { Hong Kong }\end{array}$ & $\begin{array}{l}\text { Cindy Cooper } \\
\text { UK }\end{array}$ \\
\hline $\begin{array}{l}\text { Joe Canner } \\
\text { USA }\end{array}$ & $\begin{array}{l}\text { William CS Cho } \\
\text { Hong Kong }\end{array}$ & $\begin{array}{l}\text { Philip Cooper } \\
\text { UK }\end{array}$ \\
\hline $\begin{array}{l}\text { Joseph Canner } \\
\text { USA }\end{array}$ & $\begin{array}{l}\text { Sun-Mi Choi } \\
\text { Korea, South }\end{array}$ & $\begin{array}{l}\text { Simon Coulton } \\
\text { UK }\end{array}$ \\
\hline $\begin{array}{l}\text { Toby Capstick } \\
\text { UK }\end{array}$ & $\begin{array}{l}\text { Jesper Frank Christensen } \\
\text { Denmark }\end{array}$ & $\begin{array}{l}\text { Nancy Covell } \\
\text { USA }\end{array}$ \\
\hline $\begin{array}{l}\text { Xavier Carcopino } \\
\text { France }\end{array}$ & $\begin{array}{l}\text { Robin Christensen } \\
\text { Denmark }\end{array}$ & $\begin{array}{l}\text { Peter Alan Coventry } \\
\text { UK }\end{array}$ \\
\hline $\begin{array}{l}\text { Valentina Cardi } \\
\text { UK }\end{array}$ & $\begin{array}{l}\text { Antonio Cianci } \\
\text { Italy }\end{array}$ & $\begin{array}{l}\text { Helen Cox } \\
\text { South Africa }\end{array}$ \\
\hline $\begin{array}{l}\text { Dawn Carnes } \\
\text { UK }\end{array}$ & $\begin{array}{l}\text { David Clarke } \\
\text { UK }\end{array}$ & $\begin{array}{l}\text { Susan Cox } \\
\text { Canada }\end{array}$ \\
\hline $\begin{array}{l}\text { Christopher Carroll } \\
\text { UK }\end{array}$ & $\begin{array}{l}\text { Jan Clarkson } \\
\text { UK }\end{array}$ & $\begin{array}{l}\text { Peter Craig } \\
\text { UK }\end{array}$ \\
\hline $\begin{array}{l}\text { Martin Cartwright } \\
\text { UK }\end{array}$ & $\begin{array}{l}\text { Brian Clausen } \\
\text { Denmark }\end{array}$ & $\begin{array}{l}\text { Angela Craigie } \\
\text { UK }\end{array}$ \\
\hline $\begin{array}{l}\text { Laura Castleman } \\
\text { USA }\end{array}$ & $\begin{array}{l}\text { John Cleland } \\
\text { UK }\end{array}$ & $\begin{array}{l}\text { Joanna Crocker } \\
\text { UK }\end{array}$ \\
\hline $\begin{array}{l}\text { Daiane Cerutti-Kopplin } \\
\text { Brazil }\end{array}$ & $\begin{array}{l}\text { Elisabeth Coart } \\
\text { Belgium }\end{array}$ & $\begin{array}{l}\text { Christine Cronin } \\
\text { USA }\end{array}$ \\
\hline $\begin{array}{l}\text { Xiaorong Chang } \\
\text { China }\end{array}$ & $\begin{array}{l}\text { Lizzie Coates } \\
\text { UK }\end{array}$ & $\begin{array}{l}\text { Jennifer Cunningham-Erves } \\
\text { USA }\end{array}$ \\
\hline $\begin{array}{l}\text { Robin Chatters } \\
\text { UK }\end{array}$ & $\begin{array}{l}\text { Erik Cobo } \\
\text { Spain }\end{array}$ & $\begin{array}{l}\text { Martha Curley } \\
\text { USA }\end{array}$ \\
\hline $\begin{array}{l}\text { Pei Chen } \\
\text { China }\end{array}$ & $\begin{array}{l}\text { Nicole Cockayne } \\
\text { Australia }\end{array}$ & $\begin{array}{l}\text { Geoffrey Curran } \\
\text { USA }\end{array}$ \\
\hline $\begin{array}{l}\text { Dacan Chen } \\
\text { China }\end{array}$ & $\begin{array}{l}\text { Christopher Coffey } \\
\text { USA }\end{array}$ & $\begin{array}{l}\text { Elizabeth Curtis } \\
\text { UK }\end{array}$ \\
\hline $\begin{array}{l}\text { Yuanfang Chen } \\
\text { China }\end{array}$ & $\begin{array}{l}\text { Judith Cohen } \\
\text { UK }\end{array}$ & $\begin{array}{l}\text { Alberto Dal Molin } \\
\text { Italy }\end{array}$ \\
\hline $\begin{array}{l}\text { Jianping Chen } \\
\text { Hong Kong }\end{array}$ & $\begin{array}{l}\text { Dave Collett } \\
\text { UK }\end{array}$ & $\begin{array}{l}\text { Amanda Daley } \\
\text { UK }\end{array}$ \\
\hline $\begin{array}{l}\text { Bin Cheng } \\
\text { China }\end{array}$ & $\begin{array}{l}\text { Clare Collins } \\
\text { Australia }\end{array}$ & $\begin{array}{l}\text { Philippa Dall } \\
\text { UK }\end{array}$ \\
\hline
\end{tabular}




\begin{tabular}{|c|c|c|}
\hline Rafael Dal-Ré & Martin Descarreaux & David Ellard \\
\hline Spain & Canada & UK \\
\hline Lynae Darbes & Catherine D'Este & Philipp Eller \\
\hline USA & Australia & Austria \\
\hline Elizabeth Dartnall & Jan D'Haese & Elham Emami \\
\hline South Africa & Germany & Canada \\
\hline Eric Daugas & Gianfranco Di Gennaro & Stephan Emich \\
\hline France & Italy & Austria \\
\hline Gail Davey & Laura Diamond & Sarah Engebretsen \\
\hline UK & Australia & USA \\
\hline Tracey Davidson & Neal Dickert & Steven Engebretson \\
\hline UK & USA & USA \\
\hline Geertruida H de Bock & Markus K Diener & Mert Erkan \\
\hline Netherlands & Germany & Germany \\
\hline Alan de Brauw & Benjamin Djulbegovic & Bente Appel Esbensen \\
\hline USA & USA & Denmark \\
\hline Rocco de Filippis & Peter Dodek & Munira Essat \\
\hline Italy & Canada & UK \\
\hline Renate de Jongh & Gordon Doig & Farhad Etezadi \\
\hline Netherlands & Australia & Iran \\
\hline Jorge de la Torre & Feixia Dong & Mohammed Ezzeldin \\
\hline USA & China & Spain \\
\hline Raphael de Souza & Tara Donker & Giacomo Faldella \\
\hline Brazil & Australia & Italy \\
\hline Bradley de Vries & Joseph Donnelly & Paul Farrand \\
\hline Australia & USA & UK \\
\hline Chris Deery & Harriet Downing & Barbara Farrell \\
\hline UK & UK & UK \\
\hline Daniela DeFrino & Janine Dretzke & James Feldman \\
\hline USA & UK & USA \\
\hline Brendan Delaney & Didier Dreyfuss & Natasha Fernandes \\
\hline UK & France & Canada \\
\hline Giorgio Della Rocca & Lea Drye & Frederick Ferris \\
\hline Italy & USA & USA \\
\hline Jacques Demotes & Joel Dubin & Shona Fielding \\
\hline France & Canada & UK \\
\hline Martin Dennis & Lelia Duley & Francis Finucane \\
\hline UK & UK & Ireland \\
\hline Martin Denvir & Jo Dumville & Christina Fitzner \\
\hline UK & UK & Germany \\
\hline Sonja Derman & TA Duong Dinh & Deborah Fitzsimmons \\
\hline Germany & Germany & Canada \\
\hline Timothy DeRouen & Deep Dutta & John Fletcher \\
\hline USA & India & Australia \\
\hline
\end{tabular}


Peter Fonagy

UK

Maelán Fontes Villalba

Spain

Christen Fornadel

USA

David Forster

USA

Laura Forsythe

USA

Charlie Foster

UK

Marlene Fransen

Australia

Rosanne Freak-Poli

Australia

Tobias Freund

Germany

Tim Friede

Germany

Lawrence Friedman

USA

Jordan Fulcher

Australia

Jens Gaab

Switzerland

Jakub Gajewski

Ireland

Timothy Ganey

USA

Silvio Garattini

Italy

Azucena Garcia Palacios

Spain

Hrvoje Gasparovic

Croatia

Sebastien Gibot

France

Lesley Gillespie

New Zealand

Fiona Gillison

UK

Michael Gionfriddo

USA
Timothy Girard

USA

Bruno Giraudeau

France

Anne-Marie Glenny

UK

Tove Godskesen

Sweden

Amit Goel

India

Henriette Golcher

Germany

Michael Goldfarb

Canada

Charlie Goldsmith

Canada

Tim Gomersall

UK

Javier Gonzalez

UK

José Antonio González Alastrué

Spain

Steve Goodacre

UK

Steven Goodman

USA

Philip Goodney

USA

Rebecca Gossage-Worrall

UK

Yann Goueffic

France

Norbert Graf

Germany

Nadine Graham

Canada

Aileen Grant

UK

Sean Grant

USA

Jocelyn Gravel

Canada

Celia Gregson

UK
Andrew Grieve

UK

James Griffin

UK

Xavier Griffin

UK

Francisco Gude

Spain

Hilary Gunn

UK

Kurinchi Gurusamy

UK

LB Haddad

Brazil

Terry Haines

Australia

Nigel Hall

UK

Deborah Hall

UK

Katherine Hall

USA

Daniel Hall

USA

Christopher Halloran

UK

Lisa Hampson

UK

Helen Handoll

UK

Catherine Hankey

UK

Nicola Harman

UK

Frank Harrell

USA

Kathleen Harrington

USA

Jan Hartvigsen

Denmark

Anna Hawkes

Australia

Jean Hay-Smith

New Zealand 
Liyun He
China
L Heckel
Australia
Siew Wan Hee
UK

Anna Hegedus

Switzerland

Charles Heilig

USA

Karla Hemming

UK

Ronald Henry

Netherlands

Hans-Werner Hense

Germany

Moonseong Heo

USA

Peter Herbison

New Zealand

Sarah Hetrick

Australia

Spencer Hey

Canada

Grainne Hickey

Ireland

Leanne Hides

Australia

Paul Hindmarch

UK

Allison Hirst

UK

Andrew Hoel

USA

Achim Hoerauf

Germany

John Hoey

Canada

Michael Höfler

Germany

Justus Hofmeyr

South Africa

Dan Eik Høfsten

Denmark
Rolf Holle

Germany

Ian Holloway

UK

Soren Holm

Liu Hongwei

China

Richard Hooper

UK

Dell Horey

Australia

Kim Houlind

Denmark

Michael Howley

USA

Yen-Hsuan Hsu

Taiwan

Xi-Chun $\mathbf{H u}$

China

Gill Hubbard

UK

Adwoa Hughes-Morley

UK

John Hustad

USA

Melinda Hutchesson

Australia

Cathy Hutchison

UK

Wataru Ichikawa

Japan

Olubukola Idoko

Gambia

Joanne Ingwall

USA

Talia Isaacs

UK

Shariful Islam

Australia

Khalida Ismail

UK

Saki Ito

USA
Patricia Jabre

France

Rod Jackson

New Zealand

Insoo Jang

Korea, South

Mark Jayes

UK

Micoulaud Franchi Jean-Arthur

France

Gerald Jerome

USA

Guang Ji

Ghana

Fei Jiang

USA

Yu Jiang

USA

Bernd Jilma

Austria

Kent Johnson

Australia

Derek Johnson

USA

Kate Jolly

UK

Patricia Jones

USA

Maris Jones

USA

Sue Jordan

UK

Harald Thune Jorstad

Netherlands

Samir Joshi

India

Rohina Joshi

Australia

Andrew Judge

UK

Jon Jureidini

Australia

Suneetha Kadiyala

UK 
Yega Kalairajah

UK

Thomas Kaley

USA

Lisa Kammerman

USA

Tammy Kammin

USA

Mona Kanaan

UK

Jothy Kandasamy

UK

Didem Karadibak

Turkey

Steve Karas

USA

Igor Karp

Canada

Goulnar Kasymjanova

Canada

Artur Katz

Brazil

Jay Katz

USA

Thomas Kaulhausen

Germany

Carol Keane

Australia

Richard Keefe

USA

Patrick Kelley

USA

Deanna Kelly

USA

Joanne Kemp

Australia

Stacey Kenfield

USA

Anne Kennedy

UK

Cassandra Kenning

UK

Samantha Keogh

Australia
Sally Kerry

UK

Martin Keszler

USA

Jyoti Khadka

Australia

Yoon Bum Kim

Korea, South

Hee-Soo Kim

Korea, South

Tae-Hun Kim

Korea, South

David Kimhy

USA

Bo Juel Kjeldsen

Denmark

Annet Kleiboer

Netherlands

Tobias Kleinjung

Switzerland

Shahnaz Klouche

France

Phillip Knebel

Germany

Emily Knight

Canada

Kristian Kniha

Germany

Robin Kok

Netherlands

Esther Kok

UK

Bo Kong

Germany

Andre Konski

USA

Daniel Kotz

Netherlands

Gabriel Krastl

Germany

Tobias Krieger

Switzerland

Anton Krige

UK
Lewis Kuller

USA

Merethe Kumle

Norway

Seungwon Kwon

Korea, South

Christopher Labos

Canada

Eduardo Lagomarsino

Argentina

Markus Laimer

Switzerland

Leslie Laing

Canada

Pietro Lampertico

Italy

Athene Lane

UK

Roger Langford

UK

Jost Langhorst

Germany

Li Xing Lao

Hong Kong

Sarah Larney

Australia

Marissa Lassere

Australia

Jorgen Lauridsen

Denmark

Jens Lauritsen

Denmark

Maurice Laville

France

Julia Lawton

UK

Jimmy Le

USA

M Le Guen

France

Joanna Leaviss

UK

Robert Lebeau

USA 
Annie LeBlanc

USA

Myeong Soo Lee

Korea, South

Seung Eun Lee

Korea, South

Minji Lee

USA

Jun-Hwan Lee

Korea, Republic of

Chii-Ming Lee

Taiwan

Paul Lee-Archer

Australia

Jungtae Leem

Korea, South

Francois Lemaire

France

Gilles Lemesle

France

Dennis Lendrem

UK

Emmanuel Lesaffre

Belgium

Kate Leslie

Australia

Steff Lewis

UK

Xinyan Li

China

Min Li

Hong Kong

Ping Li

China

Yi-Heng Li

Taiwan

Shaojung Li

Taiwan

Fan-Rong Liang

China

Chien-Chang Liao

Taiwan

Richard Liebano

Brazil
Richard Lilford

UK

Zhixiu Lin

Hong Kong

Yang-Hua Lin

Taiwan

Augusto Litonjua

USA

Cun-Zhi Liu

China

Jian-Ping Liu

China

Zhihong Liu

China

Lizhou Liu

New Zealand

Teresa Liu-Ambrose

Canada

Christopher Lo

Canada

Dileep Lobo

UK

Carl Lombard

South Africa

Dustin Long

USA

Kirsty Loudon

USA

Chuanjian Lu

China

Elizabeth Lutge

South Africa

Jinhui Ma

Canada

Graeme MacLennan

UK

Julia Mader

Austria

Michael Maes

Australia

Lisa Maguire

UK

Dermot Maher

Switzerland
Govind Makharia

India

Khurram Malik

USA

Laxmaiah Manchikanti

USA

Laurent Mandelbrot

France

Ulrich Mansmann

Germany

Lamberto Manzoli

Italy

Louise Maranda

USA

Branka Marinovic

Croatia

Amelia Marques

Brazil

Zoe Marshman

UK

Seth Martin

USA

Marrissa Martyn-St James

UK

Mihaela Matei

France

Yoichi Matsui

Japan

Luciana Matsutani

Brazil

Richard Mattick

Australia

Gian Carlo Mattiucci

Italy

Nancy Mayo

Canada

Craig McBride

Australia

Corrigan McBride

USA

Catriona McDaid

UK

Patrick McEwan

USA 
Aoife McGarvey

Australia

Mark McGovern

USA

Alison H McGregor

UK

Brian McGuire

Ireland

Amanda McIntyre

Canada

Christopher McKevitt

UK

Noreen Mdege

UK

Arianeb Mehrabi

Germany

Roberto Méndez-Sánchez

Spain

Sarah Meneses

Brazil

Alain Mercat

France

Hanna Merk

Sweden

Sally Merry

New Zealand

Luc Mertens

Canada

David Metcalfe

UK

William Meurer

USA

Pierre-Luc Michaud

Canada

Nicola Mills

UK

Yosuke Miyashita

USA

Somaia Mohamed

USA

Stephen Möhlhenrich

Germany

Ben Willem Mol

Netherlands
Helen Monaghan

Australia

Toni Monleon

Spain

Leonard Montenij

Netherlands

In Seok Moon

Korea, South

Sarah Moore

Switzerland

Iain Moppett

UK

Mariana Moreira da Silva

Brazil

Gemma Morgan

UK

William Mortenson

Canada

Othmar Moser

Germany

Loren Mowszowski

Australia

Thomas Mücke

Germany

Mark Mullee

UK

Keith Muller

USA

Praveen Mummaneni

USA

Gordon Murray

UK

David Musch

USA

Charles Nager

USA

Mor Nahum

USA

Madhavan Nair

India

Zoher Naja

Lebanon

Mohammad Javad Nasiri

Iran
Helene Korvenius Nedergaard

Denmark

Melissa Neuman

UK

Corine Awonja Ngufor

UK

Michael Nicholson

UK

Marc Nickels

Australia

Massimo Nicolo'

Italy

Remy Nizard

France

Devon Noonan

USA

Gill Norman

UK

Seth Norrholm

USA

Saad Nseir

France

Kevin OBrien

UK

Ayodele Odutayo

Canada

Akiko Okifuji

USA

Sjurdur Olsen

Denmark

Stefano Omboni

Italy

Robert O'Neill

USA

Albert Oriol

Spain

Anthony Ormerod

UK

Augustine Osman

USA

Katja Ott

Germany

Petronella Ottevanger

Netherlands 
Nathan Leon Pace

USA

Salvatore Paiella

Italy

Montse Palacio

Spain

Rebecca Palmer

UK

Amanda Palmer

USA

Angeliki Papadaki

UK

Sophia Papadakis

Canada

Kosmas Paraskevas

Greece

Martyn Parker

UK

Christopher Parshuram

Canada

Nick Parsons

UK

Sujata Patil

USA

Oliver Peacock

UK

Elizabeth Pearce

USA

Dominic Pearson

UK

Claudia Pedroza

USA

Jose Penalvo

USA

Wenceslao Penate

Spain

Stephen Pereira

UK

Nuria Perez-Alvarez

Spain

Irene Pericot-Valverde

Spain

Oliver Perkin

UK
Julien Peron

France

Sean Perrin

UK

Elodie Perrodeau

France

Daniel Christopher Perry

UK

Florian Peters

Germany

Ron Peters

Netherlands

Andrew Peterson

USA

Phillip Peterson

USA

Mila Petrova

UK

Lira Pi

USA

Gilda Piaggio

France

Mirjana Pibernik-Okanovic

Croatia

Jay Piccirillo

USA

Gisele Pickering

France

Stefan Pilz

Austria

Romain Pirracchio

USA

Marie Pitkethly

UK

Robert Platt

Canada

Jan Poelaert

Belgium

Tamara Poljicanin

Croatia

Robert Pollard

USA

Gregory Pond

Canada
Caridad Pontes

Spain

Raphael Porcher

France

Colin Powell

UK

Melanie Prague

USA

Andrew Prayle

UK

Jennifer Preston

UK

Melanie Price

Australia

Lindsay Prior

UK

Pascal Probst

Germany

Riccardo Proietti

Italy

Audrey Prost

UK

John Prowle

UK

Kristi Pruiksma

USA

Livia Puljak

Croatia

Nithima Purepong

Thailand

Im Quah-Smith

Australia

Jean-Pierre Quenot

France

Terence Quinn

UK

Terry Quinn

UK

Virginia Quinn

USA

Praveer Rai

India

Nicole Rankin

Australia 
Madhumathi Rao

USA

Tim Rapley

UK

Petra Rauchhaus

UK

David Ray

UK

Julie Redfern

Australia

Stefania Redolfi

France

George Reed

USA

Barnaby Reeves

UK

Jean Marc Regimbeau

France

Jean-Philippe Regnaux

France

Jean Reignier

France

Cecile Remuzat

France

Jean-Sebastien Renaud

Canada

Suzanne Richards

UK

Jo Rick

UK

Philip Riley

UK

Robert Rintoul

UK

Jamie Roberts

USA

Jamie Roberts

USA

Thompson Robinson

UK

Helen Rodgers

UK

Isabel Rodriguez-Barraquer USA
Pierre Rompré

Canada

Nitin Roper

USA

Stephen Rosenstiel

USA

Joao Ruaro

Brazil

Julia Rucklidge

New Zealand

Belén Ruiz-Antorán

Spain

Jacqueline Rushby

Australia

Everardo Saad

Brazil

Christoph Saely

Austria

Saruta Saengtipbovorn

Thailand

Joaquín Sáez-Peñataro

Spain

Raphael Saginur

Canada

Christopher Saigal

USA

Daisuke Sakai

Japan

Brodie Sakakibara

Canada

Ian Saldanha

USA

Gil Salles

Brazil

Nir Salomon

Israel

Judith Samonigg

Austria

Arantxa Sancho-Lopez

Spain

Joseph Sanford

USA

Karla Santo

Australia
Sandra Saperstein

USA

Jan M Sargeant

Canada

David Saunders

Thailand

Benedicte Sautenet

France

Dirk Schaedler

Germany

Roberta Scherer

USA

Michael Schlussel

UK

Thomas Schmidt

Germany

Marguerite Schneider

South Africa

Andreas Schnitzbauer

Germany

Rob Scholten

Netherlands

Vanessa Scholtes

Netherlands

David Schriger

USA

Peter Schwarz

Germany

Falk Schwendicke

Germany

Philip Sedgwick

UK

Paul Seed

UK

Hanna Seidling

Germany

Patrick Sequeira-Byron

Switzerland

David Shade

USA

Haleema Shakur

UK

Hongcai Shang

China 
Surya Kant Sharma

India

Virginia Sharpe

USA

Zeeshan Sheikh

Canada

Johannah Shergis

Australia

Jian-Rong Shi

China

Maurice Sillen

Netherlands

Iveta Simera

UK

Greg Simon

USA

Ian Sinha

UK

Trygve Skonnord

Norway

Susan Slaughter

Canada

Nicola Small

UK

Valerie Smith

Ireland

Scott Smith

USA

Claire Snowdon

UK

Ameenat Lola Solebo

UK

Daniel Solomon

USA

Harald Sourij

Austria

Manon Spaander

Netherlands

Anne Speckens

Netherlands

Dominique Sprumont

Switzerland

Vinit Srivastava

India
Philip Stahel

UK

Nigel Stallard

UK

Zeno Stanga

Switzerland

Alisa Stephens Shields

USA

Fiona Stewart

UK

Mette Jensen Stochkendahl

Denmark

Jennifer Straatman

Netherlands

Daniel Strech

Germany

Stacey Su

USA

Carolyn Summerbell

UK

Yan Sun

China

Wei-Zen Sun

Taiwan

Hulya Sungurtekin

Turkey

Christina Surawy

UK

Cynthia Swarnalatha

Canada

Stephen Swisher

USA

Matthew Robert Sydes

UK

Monica Taljaard

Canada

Patricia Tang

Canada

David Tappin

UK

Martin Tauschmann

UK

Adrian Taylor

UK
Inna Tchivileva

USA

Ekmel Tezel

Turkey

Hiran Thabrew

New Zealand

Jay Thakkar

Australia

George Thanassoulis

Canada

Achilleas Thoma

Canada

Lucy Thomas

Australia

Simon Francis Thomsen

Denmark

Margaret Thorogood

UK

Kevin Thorpe

Canada

Lene Thorsen

Norway

Svetlana Tikhonova

Canada

Nickolai Titov

Australia

Angela Todd

Australia

Susan Todd

UK

Merran Toerien

UK

Palle Toft

Denmark

George Tomlinson

Canada

David Torgerson

UK

Ferran Torres

Spain

Anna Towers

Canada

Viet-Thi Tran

France 
Amy Traylor

USA

Shaun Treweek

UK

Lazaros Tsalikis

Greece

Lucy Turner

Canada

James Turner

UK

Chris Twelves

USA

Jos Twisk

Netherlands

Nikolaos Tzemos

UK

Jacob Udell

Canada

Gerard Urrutia

Spain

Antonis Valachis

Sweden

Johanna van der Bom

Netherlands

Rieke van der Graaf

Netherlands

Martinus Hendrikus Gerardus Maria van der Pas

Netherlands

Hidde P van der Ploeg

Netherlands

Bart van der Worp

Netherlands

Jeroen van Meijgaard

USA

Talitha Verhoef

UK

Helena Verkooijen

Netherlands

Renee Verwey

Netherlands

Andrew Vickers

USA
Mauro Vigan Ograve

Italy

Anna Virgili

Italy

Benno Von Bormann

Thailand

Alexander Vonbank

Austria

Kalpana Vora

India

Francois Vrtovsnik

France

Helen Wakeford

UK

Jens Waldmann

Germany

Peter Wall

UK

Sherrie Wallington

USA

Shu Wang

China

Jaw-Yuan Wang

Taiwan

James Wason

UK

Heather Waterman

UK

Fiona Webster

Canada

Ruth Webster

Australia

Odette Wegwarth

Germany

Charles Weijer

Canada

Christel Weiss

Germany

Carolina Weller

Australia

Patrick Wen

USA
Zehuai Wen

China

Keith Wheatley

UK

Angela White

Canada

Peter White

UK

Will Whiteley

UK

Patricia Whitfield

New Zealand

Christian Wiedermann

Italy

Lucas Wiegand

USA

Phil Wiffen

UK

Sally Louise Williams

Italy

Don Willison

Canada

Paul M Wilson

UK

Anne Wilson

UK

Jennifer Wingham

UK

Zoe Winters

UK

Michelle Wise

New Zealand

Nicholas Wise

USA

Charles Wiysonge

South Africa

Barbara Wizner

Poland

Janice Wong

USA

Kerry Woolfall

UK 


\begin{tabular}{|c|c|c|}
\hline $\begin{array}{l}\text { Bo Wu } \\
\text { China }\end{array}$ & $\begin{array}{l}\text { D Yates } \\
\text { UK }\end{array}$ & $\begin{array}{l}\text { Ping Zhao } \\
\text { China }\end{array}$ \\
\hline $\begin{array}{l}\text { Justin Wu } \\
\text { Hong Kong }\end{array}$ & $\begin{array}{l}\text { Amelie Yavchitz } \\
\text { France }\end{array}$ & $\begin{array}{l}\text { Ling Zhao } \\
\text { China }\end{array}$ \\
\hline $\begin{array}{l}\text { Yen-Wen Wu } \\
\text { Taiwan }\end{array}$ & $\begin{array}{l}\text { Bridget Young } \\
\text { UK }\end{array}$ & $\begin{array}{l}\text { Cuihong Zheng } \\
\text { China }\end{array}$ \\
\hline $\begin{array}{l}\text { Qian Wu } \\
\text { USA }\end{array}$ & $\begin{array}{l}\text { Jing Yuan } \\
\text { Hong Kong }\end{array}$ & $\begin{array}{l}\text { Linda LD Zhong } \\
\text { Hong Kong }\end{array}$ \\
\hline $\begin{array}{l}\text { Rudolf P Wüthrich } \\
\text { Switzerland }\end{array}$ & $\begin{array}{l}\text { Nese Yuksel } \\
\text { Canada }\end{array}$ & $\begin{array}{l}\text { Linda Zhong } \\
\text { Hong Kong }\end{array}$ \\
\hline $\begin{array}{l}\text { Jielai Xia } \\
\text { China }\end{array}$ & $\begin{array}{l}\text { Joanna Zakrzewska } \\
\text { UK }\end{array}$ & $\begin{array}{l}\text { Fen Zhou } \\
\text { China }\end{array}$ \\
\hline $\begin{array}{l}\text { Bin Xu } \\
\text { China }\end{array}$ & $\begin{array}{l}\text { Ann Zauber } \\
\text { USA }\end{array}$ & $\begin{array}{l}\text { Bingmei Zhu } \\
\text { China }\end{array}$ \\
\hline $\begin{array}{l}\text { Kyohei Yamaji } \\
\text { Japan }\end{array}$ & $\begin{array}{l}\text { William Zempsky } \\
\text { USA }\end{array}$ & $\begin{array}{l}\text { Feng-Cai Zhu } \\
\text { China }\end{array}$ \\
\hline $\begin{array}{l}\text { Yong-Qing Yang } \\
\text { China }\end{array}$ & $\begin{array}{l}\text { Pamela Zengel } \\
\text { Germany }\end{array}$ & $\begin{array}{l}\text { Jose Zubizarreta } \\
\text { USA }\end{array}$ \\
\hline $\begin{array}{l}\text { Jie Yang } \\
\text { China }\end{array}$ & $\begin{array}{l}\text { Yong Zhang } \\
\text { China }\end{array}$ & $\begin{array}{l}\text { Massarat Zutshi } \\
\text { USA }\end{array}$ \\
\hline $\begin{array}{l}\text { Zifeng Yang } \\
\text { China }\end{array}$ & $\begin{array}{l}\text { Qinhong Zhang } \\
\text { China }\end{array}$ & $\begin{array}{l}\text { Merrick Zwarenstein } \\
\text { Canada }\end{array}$ \\
\hline $\begin{array}{l}\text { Mingxiao Yang } \\
\text { China }\end{array}$ & $\begin{array}{l}\text { Zhigang Zhang } \\
\text { China }\end{array}$ & \\
\hline
\end{tabular}

